\title{
A Problem in Self-Heating of a Spherical Body
}

\author{
S. M. Genensky*
}

\begin{abstract}
An analytic steady-state solution is developed for a spherical body in which heat is generated according to a first-order unimolecular-reaction law, and lost at the surface in accordance with Newton's law of cooling. The temperature within the sphere depends chiefly on the radial distance from the center, but also on the ambient temperature, the surface heat-loss coefficient, and the material properties, which are assumed constant.
\end{abstract}

\section{Introduction}

The analysis described was pursued in connection with an investigation into the self-heating of fibrous materials carried on at the National Bureau of Standards. ${ }^{1}$

Comparison of the results of this analysis with the experimental results indicated that the assumption of a single first-order unimolecular reaction was an oversimplification for the material investigated experimentally. However, the analysis serves to indicate the relative importance of the various parameters entering into the problem, and may be helpful in indicating the kind of data that would be of greatest use in further investigations in which the assumptions may be applicable.

A homogeneous sphere of radius $B$ is generating heat, under steady-state conditions, in accordance with a first-order unimolecular-reaction law, $A e^{-(E / R T)}$, and losing heat from its surface to an atmosphere at constant temperature, $T_{a}$, according to Newton's law of cooling. The temperature of the sphere, $T(r)$, is independent of time and depends upon the radial distance, $r$, from the center of the sphere. Within the sphere, transfer of heat by means other than conduction is considered negligible. The thermal conductivity, $k$; gas constant, $R$; rate of reactionfrequency product, $A$; and activation energy, $E$, of the material of the sphere are assumed to be known constants, as are the temperature, $T_{0}$, and gradient $d T / d r=0$, at the center of the sphere. The problem is to find the surface temperature of the sphere, $T_{B}$, at $r=B$, and the heat-transfer coefficient, $h$, between the surface of the sphere and a surrounding atmosphere.

\section{Analysis}

Mathematically the problem becomes

$$
\begin{gathered}
k\left[\frac{d^{2} T}{d r^{2}}+\frac{2}{r} \frac{d T}{d r}\right]+A e^{-\frac{E}{R T}}=0 \quad(0<r<B), \\
T=T_{0} \quad \text { at } \quad r=0,
\end{gathered}
$$

*Present address: Brown University, Providence, R. I. 1 N. D. Mitchell, New light on self-ignition, Natl. Fire Protect. Assoc. Quart. (October 1951).

$$
\begin{gathered}
\frac{d T}{d r}=0 \quad \text { at } \quad r=0, \\
k\left(\frac{d T}{d r}\right)_{r=B}=-h\left[T_{B}-T_{a}\right] .
\end{gathered}
$$

Let

$$
\eta=\frac{r}{B}, \quad V(\eta)=\frac{R}{E} T(\eta), \quad \text { and } \quad C=\frac{A R B^{2}}{E k} \text {. }
$$

Then eq (1) to (4) become

$$
\begin{gathered}
\frac{d^{2} V}{d \eta^{2}}+\frac{2}{\eta} \frac{d V}{d \eta}+C e^{-\frac{1}{V}}=0 \quad(0<\eta<1), \\
V=V_{0}=\frac{R T_{0}}{E} \quad \text { at } \quad \eta=0, \\
\frac{d V}{d \eta}=0 \quad \text { at } \quad \eta=0, \\
k\left(\frac{d V}{d \eta}\right)_{\eta=1}=-\frac{B h R}{E}\left[T_{B}-T_{a}\right] .
\end{gathered}
$$

It is further assumed that (a) the temperature gradient within the sphere exists and is continuous throughout the interval $0 \leq \eta \leq 1$, and (b) that the absolute value of the difference between $T(\eta)$, $0 \leq \eta \leq 1$, and $T_{0}$ is small in comparison to $T_{0}$. Under these restrictions, a technique presented by Chambre ${ }^{2}$ proves useful to observe that

$$
\frac{1}{V}=\frac{1}{V_{0}-\Delta V}=\frac{1}{V_{0}}\left[1 /\left(1-\frac{\Delta V}{V_{0}}\right)\right]=\frac{1}{V_{0}} \sum_{i=0}^{\infty}\left(\frac{\Delta V}{V_{0}}\right)^{i},
$$

where $\Delta V=V_{0}-V$. Now, because $\left|\Delta V / V_{0}\right|<1$, the series converges, and for a sufficiently large positive integer, $N,\left(1 / V_{0}\right) \sum_{i=0}^{N}\left(\Delta V / V_{0}\right)^{i}$ is a very good approximation to $1 / V$.

Therefore, (5) may be written approximately as

$$
\frac{d^{2} V}{d \eta^{2}}+\frac{2}{\eta} \frac{d V}{d \eta}+C e^{-\frac{1}{V_{0}} \sum_{i=0}^{N}\left(\frac{\Delta V}{V_{0}}\right)^{i}}=0 \quad(0<\eta<1) .
$$

${ }^{2}$ P. L. Chambré, On the solution of the Poisson-Boltzman equation with application to the theory of thermal explosions, J. Chem. Phys. 20, 1795 (1952). 
Now

$\sum_{i=0}^{N}\left(\frac{\Delta V}{V_{0}}\right)^{i}=\sum_{i=0}^{N}\left(1-\frac{V}{V_{0}}\right)^{i}=(N+1)-\boldsymbol{B}_{1} V+\boldsymbol{B}_{2} V^{2}$

where

$$
\boldsymbol{B}_{k}=\sum_{n=0}^{N} \frac{n !}{(n-k) ! k ! V_{0}^{k}}, \quad k=1,2, \ldots, N,
$$

and in the special case $V=V_{0}$

$$
\begin{array}{r}
(N+1)-\boldsymbol{B}_{1} V+\boldsymbol{B}_{2} V^{2}-\ldots+(-1)^{N} \boldsymbol{B}_{N} V^{N}=1 \\
N=0,1,2, \ldots
\end{array}
$$

Therefore, (10) may be written

$\frac{d^{2} V}{d \eta^{2}}+\frac{2}{\eta} \frac{d V}{d \eta}+C e^{-\frac{N+1}{V_{0}}} e^{\frac{1}{V_{0}}\left[\boldsymbol{B}_{1} V-\boldsymbol{B}_{2} V^{2}+\ldots-(-1)^{N} \boldsymbol{B}_{N} V^{N}\right]}=0$.

Because (11) is analytic and regular except at $\eta=0$, and further as $d V / d \eta=0$ at $\eta=0$, the solution of (11) may be written in the form

$$
V=V(\eta)=\sum_{i=0}^{\infty} a_{i} \eta^{i} \quad(0 \leq \eta \leq 1) .
$$

Recaliing (6), eq (12) at $\eta=0$ yields

$$
V_{0}=V(0)=\frac{T_{0} R}{E}=a_{0},
$$

and further differentiating (12) once and considering (7), it is found that

$$
a_{1}=0 \text {. }
$$

Differentiating (12) twice, substituting these first two derivatives into eq (11) and rearranging terms:

$$
\sum_{i=2}^{\infty}\left(i^{2}+i\right) a_{i} \eta^{i-2}=-D e^{\frac{\boldsymbol{n}_{1}}{V_{0}}\left[\boldsymbol{B}_{1} V-\boldsymbol{B}_{2} V^{2}+\ldots-(-1)^{N} \boldsymbol{B}_{N} V^{N}\right]},
$$

where $D=C e^{-\frac{N+1}{V_{0}}}$ and the $a_{i}$ are given by

$a_{i}=-\frac{1}{\left(i^{2}+i\right)} \frac{1}{(i-2) !} \frac{d^{i-2}\left[D e^{\frac{1}{V_{0}}\left(\boldsymbol{B}_{1} V-\boldsymbol{B}_{2} V^{2}+\ldots-(-1)^{N} \boldsymbol{B}_{N} V^{N}\right)}\right]}{d \eta^{i-2}}$

for $\eta=0$, and $i=2,3,4, \ldots$

Using the coefficients obtained from (13), (14), and (16) and substituting them into eq (12), $V$ can be computed for $0 \leq \eta \leq 1$.

In particular, for $\eta=1$, eq (12) gives the dimensionless surface temperature $V(1)$, and thus recalling that $T(\eta)=(E / R) V(\eta)$, the surface temperature, $T(1)$, which equals $T_{B}$, is easily found. Differentiating eq (12) once and evaluating this derivative at $\eta=1$, $h$ can then be found by solving eq (8), for all the other factors involved in this equation are either given or have been computed.
The first six nonzero coefficients have been evaluated and are

$a_{0}=V_{0}$

$a_{2}=-\frac{C}{6} e^{-\frac{1}{V_{0}}}$

$a_{4}=\frac{C^{2}}{120 V_{0}^{2}} e^{-\frac{2}{V_{0}}}$

$a_{6}=\frac{C^{3}}{1008 V_{0}^{3}}\left[\frac{8}{15} \frac{C}{V_{0}}-\frac{2}{3}\right] e^{-\frac{3}{V_{0}}}$

$a_{8}=\frac{C^{4}}{51840 V_{0}^{4}}\left[\frac{122 C^{2}}{63 V_{0}^{2}}-\frac{122 C}{21 V_{0}}+\frac{10}{3}\right] e^{-\frac{4}{V_{0}}}$

$a_{10}=\frac{C^{5}}{4435200 V_{0}^{5}}\left[\frac{5032 C^{3}}{405 V_{0}^{3}}-\frac{8428 C^{2}}{135 V_{0}^{2}}+\frac{1486 C}{27 V_{0}}-\frac{560}{9}\right] e^{-\frac{5}{V_{0}}}$

Observe that $a_{2 i-1}(i=1,2,3, \ldots)$ are zero. This follows from $a_{1}=0$ and the spherical symmetry of the problem.

Figure 1 is a plot of $V(1)$, as a function of $C$ and $1 / V_{0}$, where $C$ was allowed to vary over the range $10^{-1} \leq C \leq 10^{19}$ and $1 / V_{0}$ took on selected values in the range $5 \leq 1 / V_{0} \leq 100$. The points were obtained by using

$$
V(\eta)=\sum_{i=0}^{10} a_{i} \eta^{i}
$$

over its range of applicability for the values of $C^{t}$ and $1 / V_{0}$ within the above limits.

In the foregoing, it has been assumed that the temperature at the center of the sphere $T_{0}$ is known, and also that the physical condition indicated by eq (8) is satisfied.

The more common steady-state problem of finding the temperature at the center of a sphere that generates heat according to a first-order unimolecular reaction law and loses heat according to Newton's law of cooling may be solved by using the analysis described above. In this case, $E, R, k, A, h, T_{a}$, $T_{B}$, and $B$ are assumed known.

If the sphere being considered really satisfies the assumptions of the problem, then its temperature must satisfy eq (1). Because eq (1) is of the second order, only two boundary conditions can be preassigned. Further, because the temperature at the center of the sphere must remain finite, one of these preassigned boundary conditions must be $d T / d r=0$ at $r=0$. This leaves but one free boundary condition. However, at the surface of the sphere both

$$
\left(\frac{d T}{d r}\right)_{r=B}=-\frac{h}{k}\left[T_{B}-T_{a}\right]
$$

and

$$
T=T_{B} \quad \text { at } \quad r=B
$$

must be satisfied; and because only one of these two 


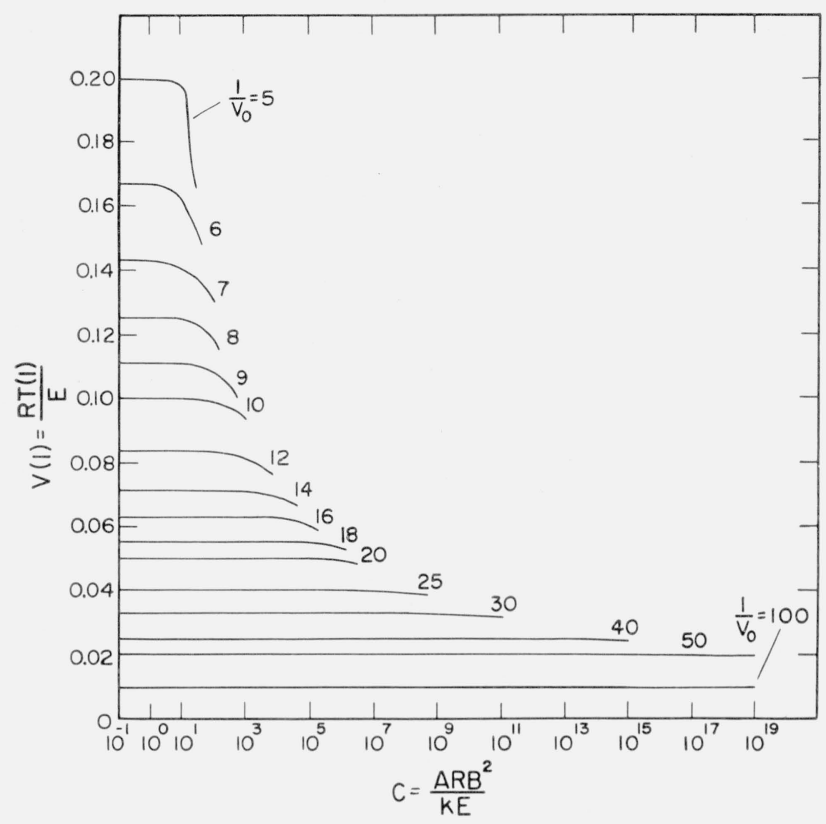

Figure 1. $\quad V(1)$ as a function of $C$ for selected values of $1 / V_{0}$. boundary conditions may be formally assigned, it must be assumed that the other is consistent with the problem. Therefore, figure 1, which represents eq (17), may be used to solve this problem. Because $R, E, k, A$, and $B$ are known, both $C$ and $V(1)$ (recall that $T_{B}=E V(1) / R$ ) can be computed. Thus, by using figure $1,1 / V_{0}$ may be found by interpolation, provided the point $(C, V(1))$ under consideration lies within the region of applicability of eq (17), as indicated above. Because the center temperature of the sphere is $T_{0}$, that is $E V_{0} / R$, the problem is solved.

Here no use was made of condition (18), and as mentioned earlier, this condition must be satisfied independently, if the assumptions made are fulfilled. Thus the degree of consistency between conditions (18) and (19) serves as an indication of the applicability of the assumptions made in this analysis.

Washington, March 19, 1954. 